\title{
Editorial: Symbiosis in a Changing Environment
}

\author{
Anne Duplouy ${ }^{1,2 *}$, Bradley R. Dotson ${ }^{2,3}$, Michele K. Nishiguchi ${ }^{4}$ and César A. Cárdenas ${ }^{5}$ \\ ${ }^{1}$ Organismal and Evolutionary Biology Research Program, University of Helsinki, Helsinki, Finland, ${ }^{2}$ Department of Biology, \\ Lund University, Lund, Sweden, ${ }^{3}$ Department of Integrated Plant Protection, Swedish University of Agricultural Sciences, \\ Alnarp, Sweden, ${ }^{4}$ Department of Molecular and Cell Biology, University of California, Merced, Merced, CA, United States, \\ ${ }^{5}$ Departamento Científico, Instituto Antártico Chileno, Punta Arenas, Chile
}

Keywords: environmental changes and stressors, holobiont, host-microbe associations, marine life, terrestrial life

\section{Editorial on the Research Topic}

\section{Symbiosis in a Changing Environment}

Symbiotic interactions are formed by the long-term intimate association of microorganisms and their host species. Such interactions are ubiquitous throughout the tree of life, and research suggests that they might have facilitated major evolutionary transitions and possibly the success of life on Earth (Moran, 2006, 2007; Raina et al., 2018; Kolodny et al., 2020). Symbionts owe their success to their ability to be sometimes "good" and sometimes "evil" (Jiggins and Hurst, 2011; Brannon and Mulvey, 2019; Newton and Rice, 2020). By manipulating their hosts' life history traits, fecundity, dispersal ability, and/or resistance to stresses caused by pathogens and environmental changes, these "Jekyll-and-Hyde" symbionts are promoting their own selfish fitness. The diversity of symbiont-induced phenotypes, the complexity of symbiotic interactions, their ecology and evolution through time and across environments has therefore unsurprisingly attracted the interests of a large scientific community. Under the current global context of fast environmental changes, one can predict that new and/or less predictable abiotic and biotic stressors will affect symbiotic interactions with potential cascading effects on the eco-evolutionary population dynamics of host species and the communities in which they are embedded (Pita et al., 2018; Trevathan-Tacket et al., 2019; Greenspan et al., 2020; Kolodny and Schulenburg, 2020).

Our Research Topic on Symbiosis in a Changing Environment aimed to bring together review articles, providing analyses of the state-of-the-art in the field, and experimental research articles, testing unique hypotheses on the interplay between host-symbiont interactions and their various environments. We called for submissions from a broad and diverse set of researchers, from international institutions worldwide ( $N=26$ institutions, 11 countries, 5 continents), to showcase their view and expertise on a range of symbiotic study systems, from the tropics to the Antarctic, from terrestrial arthropods and plants to marine invertebrates. The published articles are only a subset of the growing literature available in symbiosis research. However, the articles clearly present that a wide diversity of stressors, including changes in temperatures, in exposure to UV-radiations, or in metabolite provisioning, can severally affect symbiosis.

Based on the literature on terrestrial invertebrates and plants, Bénard et al., and Saikkonen et al., respectively, predict that by influencing the host's life-history traits and/or their host's response to natural stresses, Jekyll and Hyde symbionts can either exacerbate or ameliorate the effects of environmental stochasticity on their hosts. This is due to the essential role of microbial symbionts for ecological, evolutionary, and genetic processes in all higher organisms. Obligate symbioses can indeed turn into evolutionary traps or dead-ends, since the optimum ranges between hosts and their symbiont(s) may inevitably mismatch, whereas facultative symbioses can provide an adaptive solution to environmental changes and opportunities to novel niches. The mini-reviews agree that 
major progress has been made in (I) the description of microbial communities associated with organisms from different environments, although one can argue that unlike bacterial communities, fungal, and viral communities have been neglected, and (II) testing the role of various individual stressors on these interactions. However, much remains to be examined in understanding the organization and changes in these communities, and in their functionality for their hosts. Similarly, estimations of the adaptability of host-symbiont associations under multiple stressors, as it would be under natural conditions, remain scarce (Rondon et al.).

Carrier and Reitzel further synthesize the ecological importance of the host in the understanding of their associated symbiotic communities. They debate that, at least in echinoderms, the selection for bacterial communities occurs at the larval stage; however, the role of these selected bacteria for the larvae and/or for the adults of the same species remains unclear. Such gaps in the current knowledge is not restricted to studies on echinoderms. Numerous studies have shown the importance of the hosts' life-history strategies influencing the microbial communities, either because of changes in the host diet, hormones, or the environmental conditions faced at each life-stage (e.g., aquatic larvae vs. terrestrial adults in many insects or amphibians). Similarly, the health of individuals and species or populations can greatly affect the composition of their associated microbial community (Blanquer et al., 2016; Li et al., 2018), as pathogens replace or challenge symbiotic interactions in their hosts. Furthermore, the role of microbial communities is severely debated in some species. For example, the gut microbiota of Lepidoptera larvae is thought to be transient and non-functional, rather than resident and of any benefit to the host (Hammer et al., 2017; Duplouy et al., 2020).

One way to understand which symbiotic interactions are likely to thrive or disappear in changing environments, is through the investigation of the effects of individual and/or cumulated stressors on symbioses in the controlled conditions of laboratories. For example, Heyworth et al. investigate experimentally the responses of three facultative endosymbionts in the pea aphid (Acyrthosiphon pisum) to heat stress, and the influence on the host and an obligate symbiont (Buchnera). After exposing aphids to $38.5^{\circ} \mathrm{C}$, they show that two of the facultative symbionts helped the recovery of both host and the obligate symbiont after heat stress, thus suggesting that under climate changes, the presence of these facultative symbionts may be of benefit to the host species. However, response to long-term heat exposure, rather than to short heat bursts further need to be investigated.

The Rondon et al. article provides insights on the combined effects of physical drivers associated with climate change (seawater warming and ice scour) in shaping the microbiome of the common sponge Isodictya kerguelenensis from the Western Antarctic Peninsula. Their findings based on a multistressors laboratory experiment demonstrates that disturbance produced by icebergs may have direct impacts on the microbiome of this sponge species. In this regard, the results highlight the importance of this relationship as the effect of both stressors are expected to increase under future climate change scenarios, hence having the potential of producing effects on the holobiont balance and also on the ecosystem.

Barrera et al. present the role of foliar endophytic fungi on the performance of their host plant, the Antarctic host plant Colobanthus quitensis, under high UV-B radiation. By comparing the expression levels of genes involved in UV-B photoreception, flavonoid accumulation, and physiological stress in fungusinfected vs. uninfected plants, their study provides evidence that Antarctic endophytic fungi minimize cell damage and boost physiological performance and tolerance to UV-B radiation in $C$. quitensis. Therefore, endophytic fungi could be effective partners in the context of increased UV-B radiation in the Antarctic. Future studies should highlight whether the current trend in climate change may already be selecting for such associations in different parts of the world.

Currin-Ross et al. use Drosophila melanogaster flies exposed to different levels of iron provisioning (i.e., deficiency, optimum, and excess), and evaluate the conflict that arose from this stress between the host flies and their facultative bacterial endosymbiont Wolbachia, as both rely on iron-provision from the environment. Through metabolomic methods, they show that flies exhibit variation in their metabolism when exposed to low or high levels of iron, and that these metabolic responses are differently activated upon infection with Wolbachia. The bacterium maintains oxidative metabolism in its Drosophila host. Whether this is a typical response for the symbiont in insects or in Drosophila hosts specifically remains to be investigated.

This set of experimental studies highlight the diversity of symbiotic systems, and the diverse changes in phenotypes between different treatment groups. They produce unique knowledge acquired within the controlled conditions of laboratories that inform about why host-symbiont interactions form, and how they maintain or decline through time. Such discoveries support and/or complement the interpretation of patterns of diversity and changes in symbiont communities associated to species across space and time in the field.

Based on a 2-year field monitoring study of two coral species in Kāne'ohe Bay, Hawai'i, Matsuda et al. characterize responses of colonies after a bleaching event produced by anomalous local seawater temperatures. Their results show that the two studies species exhibited either a bleachingsusceptible phenotype (bleached) or resistant phenotype (nonbleached). Colonies of Porites compressa show greater resilience following bleaching than Montipora capitata, despite having higher bleaching prevalence and severity. This is suggesting that bleaching susceptibility is not always a good predictor of mortality after a warming event and consequent bleaching events. The authors stress the importance of including monitoring not only at the population level but also at the individual level to better understand coral susceptibility to warming and to better predict responses to future scenarios. 
Similarly, using a combination of in situ observations along with morphological and molecular approaches, van der Windt et al. study the relationship between bio-eroding sponges belonging to Clionaidae and Symbionidiaceaea from the IndoPacific. The findings of this study (I) suggest a high diversity of Symbionidiaceaea associated with bio-eroding species, and (II) highlight the potential importance of host identify and tolerance capacity to heat stress (up to $33^{\circ} \mathrm{C}$ ) to maintain symbiotic interactions in these sponges. Furthermore, these results suggest that symbiotic interactions in bio-eroding sponges may greatly differ in their adaptive capacity from symbioses reported in other marine organisms such as corals (Baker et al., 2008). Another study from a contrasting environment by Papale et al. provides new information on microbial communities associated with different Antarctic sponge species. Their comparative study of sponges collected from 30 to 271 depth at Terra Nova Bay in the Ross Sea region provides new information on the diversity and functional profiles of microbial symbiont communities associated with such highlatitude marine organisms. They also show the presence of rich bacterial communities dominated by Proteobacteria and their functional prediction analyses expand the current knowledge of sponge-associated microbial communities, suggesting key roles played by the microbiome, including antibiotic biosynthesis, degradation of aromatic compounds, and methane metabolism. As sponges are considered an important reservoir of exceptional microbial diversity and a major contributor to marine microbial diversity (Thomas et al., 2016), these few studies allow for the comparison of research in symbiotic systems from different marine regions (tropics vs. Antarctic) that will complement our

\section{REFERENCES}

Baker, A. C., Glynn, P. W., and Riegl, B. (2008). Climate change and coral reef bleaching: an ecological assessment of long-term impacts, recovery trends and future outlook. Estuar. Coast. Shelf Sci. 80, 435-471. doi: 10.1016/j.ecss.2008. 09.003

Blanquer, A., Uriz, M. J., Cebrian, E., and Galand, P. E. (2016). Snapshot of a bacterial microbiome shift during the early symptoms of a massive sponge die-off in the western Mediterranean. Front. Microbiol. 7:752. doi: 10.3389/fmicb.2016. 00752

Brannon, J. R., and Mulvey, M. A. (2019). Jekyll and Hyde: bugs with double personalities that muddle the distinction between commensal and pathogen. J. Mol. Biol. 431, 2911-2913. doi: 10.1016/j.jmb.2019. 06.014

Duplouy, A., Minard, G., and Saastamoinen, M. (2020). The gut bacterial community affects immunity but not metabolism in a specialist herbivorous butterfly. Ecol. Evol. 10, 8755-8769. doi: 10.1002/ece3. 6573

Greenspan, S. E., Migliorini, G. H., Lyra, M. L., Pontes, M. R., Carvalho, T., Ribeiro, L. P., et al. (2020). Warming drives ecological community changes linked to host-associated dysbiosis. Nat. Ecol. Evol. 10, 1557-1061. doi: 10.1038/s41558-020-0 899-5

Hammer, T. J., Janzen, D. H., Hallwachs, W., Jaffe, S. P., and Fierer, N. (2017). Caterpillars lack a resident gut microbiome. Proc. global knowledge of the role of symbionts in risks associated with global climate change scenarios in marine organisms.

The article collection from this Research Topic illustrates the already well-known ubiquitous character and the diversity of unique symbiotic systems, with a targeted focus on their responses to environmental change and stressors. These studies test just a few hypotheses, and introduce many more that remain and deserve to be investigated. Nonetheless, these studies clearly highlight that in order to fully grasp the role and responses of symbionts to environmental changes, more multidisciplinary and collaborative studies are needed, which will establish the essential mechanisms that forge the diverse associations between both marine and terrestrial hosts, their bacterial partners, and their environments.

\section{AUTHOR CONTRIBUTIONS}

$\mathrm{AD}$ and $\mathrm{CC}$ wrote the editorial. All authors reviewed and revised the manuscript.

\section{FUNDING}

$\mathrm{AD}$ was funded by a Marie Curie Sklodowska Individual Fellowship (\#790531, HostSweetHome) and by the Academy of Finland (\#328944). MN was funded by NASA-EXO 80NSSC18K1053 and the School of Natural Sciences at UC Merced. CC was supported by the Marine Protected Areas program of the Instituto Antártico Chileno (\#24 03 052). BD was funded by Svenska Forskningsrådet Formas FORMAS (\#2017-01263).
Natl. Acad. Sci. U.S.A. 114, 9641-9646. doi: 10.1073/pnas.17071 86114

Jiggins, F. M., and Hurst, G. D. (2011). Rapid insect evolution by symbiont transfer. Science 332, 185-186. doi: 10.1126/science.12 05386

Kolodny, O., Callahan, B. J., and Douglas, A. E. (2020). The role of the microbiome in host evolution. Philos. Trans. R Soc. Lond. B Biol. Sci. 375:20190588. doi: $10.1098 /$ rstb.2019.0588

Kolodny, O., and Schulenburg, H. (2020). Microbiome-mediated plasticity directs host evolution along several distinct time scales. Philos. Trans. R Soc. Lond. B Biol. Sci. 375:20190589. doi: 10.1098/rstb.201 9.0589

Li, Y. F., Yang, N., Liang, X., Yoshida, A., Osatomi, K., Power, D., et al. (2018). Elevated seawater temperatures decrease microbial diversity in the gut of Mytilus coruscus. Front. Physiol. 9:839. doi: 10.3389/fphys.2018. 00839

Moran, N. A. (2006). Symbiosis. Curr. Biol. 16, R866-R871. doi: 10.1016/j.cub.2006.09.019

Moran, N. A. (2007). Symbiosis as an adaptive process and source of phenotypic complexity. Proc. Natl. Acad. Sci. U.S.A. 104, 8627-8633. doi: $10.1073 /$ pnas.0611659104

Newton, I. L. G., and Rice, D. W. (2020). The Jekyll and Hyde symbiont: could. J. Bacteriol. 202:e00589-19. doi: 10.1128/JB.00589-19

Pita, L., Rix, L., Slaby, B. M., Franke, A., and Hentschel, U. (2018). The sponge holobiont in a changing ocean: from microbes to ecosystems. Microbiome 6:46. doi: $10.1186 / s 40168-018-0428-1$ 
Raina, J. B., Eme, L., Pollock, F. J., Spang, A., Archibald, J. M., and Williams, T. A. (2018). Symbiosis in the microbial world: from ecology to genome evolution. Biol Open 7:bio032524. doi: 10.1242/bio.032524

Thomas, T., Moitinho-Silva, L., Lurgi, M., Björk, J. R., Easson, C., Astudillo-García, C., et al. (2016). Diversity, structure and convergent evolution of the global sponge microbiome. Nat. Commun. 7:11870. doi: 10.1038/ncomms11870

Trevathan-Tacket, S. M., Sherman, C. D. H., Huggett, M. J., Campbell, A. H., Laverock, B., Hurtado-McCormick, V., et al. (2019). A horizon scan of priorities for coastal marine microbiome research. Nat. Ecol. Evol. 3, 1509-1520. doi: 10.1038/s41559-019-0999-7

Conflict of Interest: The authors declare that the research was conducted in the absence of any commercial or financial relationships that could be construed as a potential conflict of interest.
Publisher's Note: All claims expressed in this article are solely those of the authors and do not necessarily represent those of their affiliated organizations, or those of the publisher, the editors and the reviewers. Any product that may be evaluated in this article, or claim that may be made by its manufacturer, is not guaranteed or endorsed by the publisher.

Copyright $\odot 2021$ Duplouy, Dotson, Nishiguchi and Cárdenas. This is an open-access article distributed under the terms of the Creative Commons Attribution License (CC $B Y)$. The use, distribution or reproduction in other forums is permitted, provided the original author(s) and the copyright owner(s) are credited and that the original publication in this journal is cited, in accordance with accepted academic practice. No use, distribution or reproduction is permitted which does not comply with these terms. 